\title{
Tension pneumopericardium leads to cardiac tamponade during hand-assisted ventilation in patients with uremia
}

\author{
Qun Su, MD, ${ }^{a}$ Zhiying Feng, MD, ${ }^{b}$ Tong Li, MD, and Tao Jin, MD, ${ }^{\mathrm{c}}$ Hangzhou, China
}

$\Gamma$ ension pneumopericardium is a rare disease and may indeed be life threatening. It usually occurs in patients receiving assisted ventilation. We report a case of tension pneumopericardium in a patient during computer tomographic scanning. The CT scanning provides us with the necessary images during the development of tension pneumopericardium.

\section{Clinical Summary}

A 62-year-old female patient with a diagnosis of chronic renal insufficiency, uremia, renal anemia, and renal hypertension was admitted to the First Affiliated Hospital, School of Medicine, Zhejiand University on May 1, 2006. At 6 PM on May 6, the patient experienced palpitations after continuous renal replacement therapy was performed, and an atrial fibrillation was observed. After the administration of $5 \mathrm{mg}$ of diltiazem, the patient's heart rate decreased and returned to sinus rhythm. At 1:30 AM on May 7, the patient began

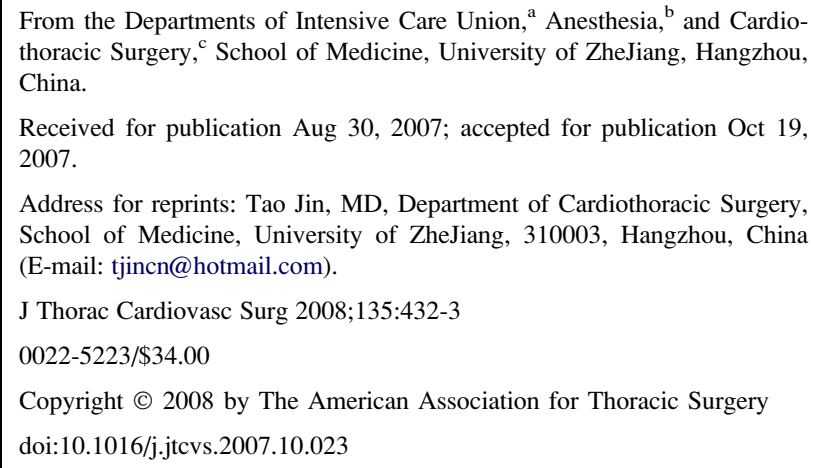

to cough blood sputum and showed chest distress and tachypnea. The life monitor showed a molecular oxygen saturation of $85 \%$ to $92 \%$, and moist rales were heard in both sides of the lung. The patient was transferred to the intensive care unit for further observation and management. Shortly afterward, the patient was intubated with mechanical ventilation support. On May 15, the intubated patient underwent a chest computed tomography (CT) scan with hand-assisted ventilation through an Ambu bag. During the examination, the patent's pulse oxygen saturation decreased dramatically and heart rate decreased gradually. Subcutaneous emphysema developed from the chest to the neck. The CT scan revealed that rightsided pneumothorax, pneumomediastinum, and tension pneumopericardium were developing (Figures 1 and 2). Two 16-gauge cannulas were punctured into both sides of the patient's chest, and compelled air was released from the right side of the chest. Epinephrin was injected intravenously, and chest compression was performed; the patient's heart rate, blood pressure, and oxygen saturation continued to decrease. Ventricular fibrillation occurred, and lidocaine was not effective. Percutaneous pericardiocentesis via the subxiphoid route was performed by the cardiac surgeon later. The patient's response was observed, but her pupils were diffused.

The patient was returned to the intensive care unit after resuscitation, but she never regained consciousness. Six hours later, the patient's condition worsened; the cardiopulmonary resuscitation procedure and further attempts were unsuccessful. Permission for autopsy was denied.

\section{Discussion}

Pneumopericardium, first described in 1844 , is defined as the presence of air in the pericardial sac. Tension pneumopericardium is a rare complication. It is defined as a pneumopericardium large enough to cause diastolic restriction and characteristic

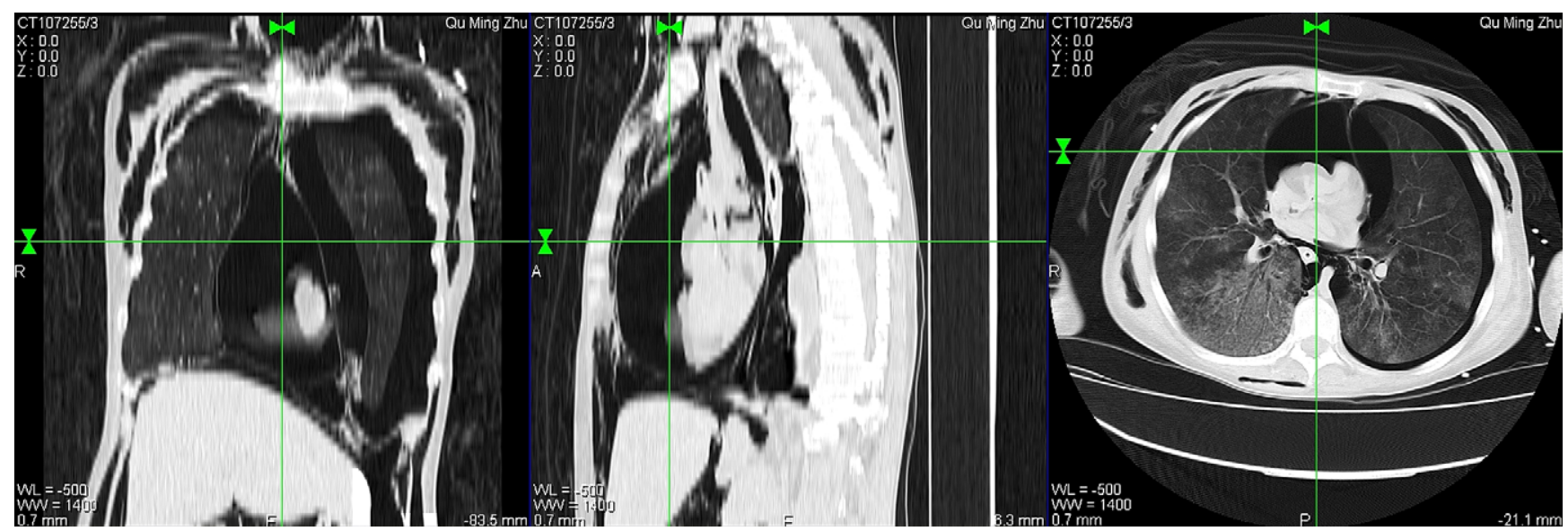

Figure 1. CT scan showing the overall view of the compressed mediastinal condition. 


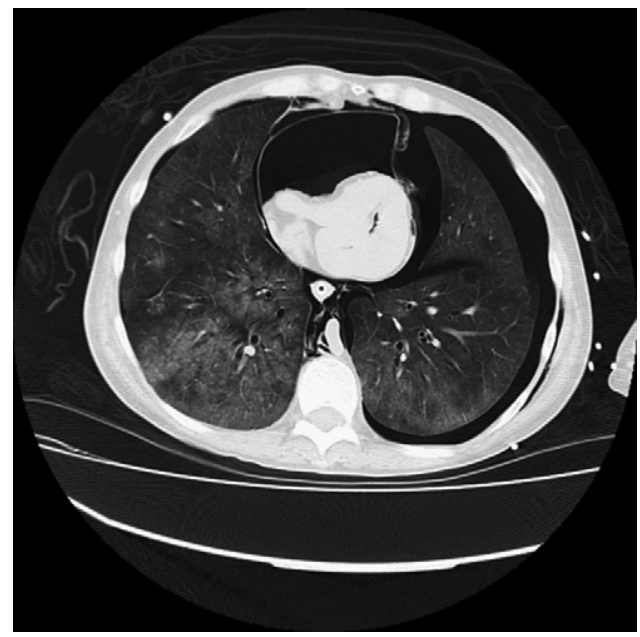

Figure 2. CT scan showing that the heart was totally compressed and that almost no room in the heart would be available for the blood.

symptoms. Pneumopericardium does not usually cause cardiac tamponade.

Various forms of ventilator assistance with high airway pressure increase the possibility of pneumopericardium, especially when there is an associated underlying pulmonary parenchymal process. ${ }^{1}$ Air under pressure ruptures the alveolar walls, dissects the blood vessels and lung segments, and eventually ruptures the mediastinal or visceral pleura, resulting in pneumomediastinum or pneumothorax. The air in turn may also enter the pericardium space at the site of the reflection of the parietal pericardium onto the visceral pericardium, producing pneumopericardium.

According to the reported cases, pneumopericardium is more apt to occur in infants than in adults. This is probably because adults have stronger adhesions between the pericardial layers, precluding communication between the pericardial space and the mediastinum. $^{2}$

In the case reported, the patient had underlying pulmonary parenchymal pathologic changes, had been intubated for 7 days, and had been ventilated with the hand-assisted Ambu bag during the
CT examination, all of which contributed to the pneumothorax, pneumomediastinum, and tension pneumopericardium, eventually leading to the cardiac tamponade.

The clinical manifestations of the tension pneumopericardium include chest pain, syncope, breathlessness, hemodynamic instability, and the auscultatory finding of a "mill-wheel" murmur arising from the movement of the heart in a pericardial cavity containing air and fluid. ${ }^{3,4}$

Radiologic imaging via a chest $\mathrm{x}$-ray or CT scan commonly identifies the presence of air in the pericardial cavity. Although pneumomediastinum and pneumopericardium are frequently confused because they can happen concomitantly, 3 signs on the chest $\mathrm{x}$-ray can help to discriminate between them: 1) a radiolucent halo of air partially or completely surrounding the heart but not extending superiorly to the attachment of pericardium, 2) a shift in pericardial air on a decubitus radiograph, and 3) the absence of a continuous diaphragm. $^{5}$

A chest CT scan is of paramount importance in the diagnosis of tension pneumopericardium. If pneumopericardium causes a cardiac tamponade, immediate pericardial decompression is required by an echocardiography-guided needle pericardiocentesis, a percutaneous drain placement, or an urgent open subxyphoid approach to the pericardium.

This was a rare case, and the CT scans were important because the tension pneumopericardium and cardiac tamponade developed during the CT examination. The salvage was unsuccessful. The reasons for the patient's death may be the anoxia and shock during the tension pneumopericardium, as well as the cardiac tamponade, which may account for the multiple organ failure.

\section{References}

1. Gammon RB, Shin MS, Groves RH, et al. Clinical risk factors for pulmonary barotrauma: a multivariate analysis. Am J Respir Crit Care Med. 1995;152:1235-40.

2. Hurd TE, Novak R, Gallagher J. Tension pneumopericardium: a complication of mechanical ventilation. Crit Care Med. 1984;12:200-1.

3. Brander L, Ramsay D, Dreier D, Peter M, Graeni R. Continuous left hemidiaphragm sign revisited: a case of spontaneous pneumopericardium and literature review. Heart. 2002;88:e5.

4. Tucker WS Jr. Symptoms and signs of syndromes associated with mill wheel murmurs. N C Med J. 1988;49:569-72.

5. McEachern RC, Patel RG. Pneumopericardium associated with facemask continuous positive airway pressure. Chest. 1997;112:1441-3. 